\title{
Unilateral versus bilateral simultaneous arthroplasties of the lower extremities
}

\author{
MICHAEL J. BUSCEMI, JR, DO \\ B.J. PAGE, II, DO \\ JOHN SWIENCKOWSKI, DO
}

We reviewed retrospectively 26 replacement arthroplasties performed as bilateral simultaneous procedures in 12 patients at an osteopathic community hospital during a 52-month period. One patient had two bilateral operations. Surgical time, estimated blood loss, units of blood transfused, perioperative morbidity, number of days hospitalized, and hospital costs were compared with data from randomly selected patients who underwent unilateral procedures during the same time period. The bilateral group had an average length of stay of 12 days (vs 10.3 days), an average surgical time of 2 hours 37 minutes (vs 1 hour 55 minutes), an average blood loss of $419 \mathrm{~mL}$ (vs $277 \mathrm{~mL}$ ), and average hospital costs of $\$ 12,315$ (vs $\$ 10,272$ ) in comparison with the unilateral group. Perioperative morbidity was similar for both groups. Our findings indicate that bilateral simultaneous procedures offer a reasonable approach and a savings of time and money for selected patients who require multiple joint replacements for improved functional status.

With the progressive refinement of total arthroplasty of the hip and knee has come increased longevity and improved functional results, thus expanding its use. Patients with bilateral involvement of the lower extremities (ie, hips and knees) will not experience full functional rehabilitation until both joints are replaced, thus broaching the feasibility of simultaneous lower extremity replacement arthroplasties under one anesthetization. The literature has well documented the advantages of one-stage bilateral total hip replacements and onestage total knee replacements ${ }^{1-11}$ with regard to fast recovery, reduced complications, decreased anesthesia time, and shortened length of hospital stay.

The purposes of this report are to delineate our perioperative protocol and to compare the postoperative morbidity, length of hospital stay, and hospital costs between patients having bilateral simultaneous lower extremity replacement arthroplasties and those having unilateral joint replacement procedures (Table).

\section{Subjects and methods}

We reviewed retrospectively the records of 12 patients who underwent 13 primary bilateral simultaneous joint replacements from January 1983 to April 1987 in an osteopathic community hospital. Five groups of patients were identified according to the surgical procedures performed: group $1 \mathrm{pa}-$ tients had bilateral total knee replacements $(\mathrm{n}=$ 3 ); group 2 underwent contralateral total hip and total knee replacements $(n=3)$; group 3 had bilateral medial unicompartmental arthroplasties of the knee $(n=4)$; group 4 had total knee and medial unicompartmental arthroplasties of the knee $(\mathrm{n}=2)$; and group 5 underwent contralateral total hip replacement and medial unicompartmental arthroplasty of the knee $(n=1)$. The data from these five groups were analyzed for statistical significance collectively and were labeled bilateral simultaneous lower extremity replacement arthroplasties.

The following components were used: the Miller Galante total knee (11), Marmor unicompartmental modular knee (11), horizontal platform supported total hip (2), and Harris Precoat total hip 
(2), for a total of 26 total joint procedures. Similarly reviewed were charts and records of 24 patients who underwent primary unilateral replacement arthroplasties during the same time period. The following procedures were performed: total hip replacement $(n=8)$, primary medial unicompartmental replacement arthroplasty of the knee $(\mathrm{n}=$ $8)$, and total knee replacement $(n=8)$. Components used were the horizontal platform supported total hip (8), Marmor unicompartmental modular knee (8), Miller Galante total knee (4), and Townley anatomic total knee (4).

\section{Surgical protocol}

In general, patients selected for bilateral arthroplasties were in good health and without substantial medical disorders. Each patient was evaluated preoperatively by an internal medicine consultant the day before surgery. Enteric-coated aspirin was administered preoperatively and continued postoperatively. Heparin, 5000 units, was given subcutaneously every 12 hours to patients who were sensitive to aspirin. Cephalosporin (first-generation) antibiotics were administered intravenously 1 hour before surgery prophylactically and up to 48 hours postoperatively. Patients highly allergic to penicillin received vancomycin preoperatively and up to 48 hours postoperatively.

Horizontal laminar airflow was used routinely. A two-team approach was used intraoperatively, including separate sets of instruments. Bilateral knee arthroplasties were worked on simultaneously by two surgical teams. For the four patients having total hip replacement and contralateral knee joint replacement, the hip initially was operated on through a lateral approach. After reducing the hip components and starting wound closure, the second team began to operate on the contralateral knee. Tourniquets were applied during knee replacement arthroplasties. After 1985, antibiotic-impregnated polymethylmethacrylate, generally in the form of gentamicin, $160 \mathrm{mg}$ per package of cement, also was used to preclude infection. ${ }^{12}$

Suction drains were used and removed generally on the second postoperative day. Antiembolic hosiery or elasticized wraps were applied from the ankle to the midthigh. Continuous passive motion machines were generally instituted by the second postoperative day. Flexion was usually increased 10 degrees per day, depending on the patient's tolerance. Patients commonly walked on the third postoperative day, with use of a walker. Physical therapy was instituted in the early postoperative period.
The hospital bill for each patient was obtained from patient accounting and reviewed. The 4-year time period of our study necessitated standardizing hospital costs and services to reflect April 1987 dollars. We used the consumer price index for hospital costs and services published by the Department of Labor, Bureau of Statistics, ${ }^{13}$ for this purpose.

A variety of components were implanted during the 4-year period in the 36 patients studied, for a total of 50 joint replacements. The cost of these components was subtracted from the total hospital bill.

Also recorded from the hospital records were estimated blood loss, surgical time, and type of anesthetic administered.

\section{Results}

\section{Bilateral group}

The average age of the 12 patients undergoing bilateral simultaneous lower extremity replacement arthroplasties, was 70 years (range, 56 to 81 years). Of the 10 women and 2 men, a preoperative diagnosis of osteoarthritis was made in 10, Paget's disease in 1 , and rheumatoid arthritis in the 1 patient who had four total joints replaced in two simultaneous bilateral sessions. Nine procedures were performed after subarachnoid block anesthesia was induced, and four operations were done with the patient under general endotracheal anesthesia.

Surgical time ranged from 1 hour 22 minutes for the bilateral Marmor medial unicompartmental knee arthroplasty group to 4 hours 26 minutes for the one-stage contralateral total hip and total knee replacement (average time, 2 hours 37 minutes).

The estimated blood loss ranged from $800 \mathrm{~mL}$ in a total knee-total hip replacement procedure to $100 \mathrm{~mL}$ for the bilateral unicompartmental knee replacement arthroplasties (average blood loss, 419 $\mathrm{mL}$ ). For $67 \%$ of the patients who received blood transfusions during their hospital stay, an average of 2 units of packed RBCs was used. Hospital length of stay ranged from 9 to 18 days (average, 12 days). The average hospital bill was $\$ 12,315$.

The one patient who was hospitalized for 18 days after a left total hip replacement and a right medial unicompartmental knee replacement arthroplasty suffered a deep vein thrombosis in the right leg. This patient was the oldest of those in the bilateral group (81 years).

Complications incurred in this bilateral group included urinary tract infection (1), superficial phlebitis (1), deep vein thrombosis (1), partial wound dehiscence secondary to hematoma (1), and transi- 
tory confusion, which resolved without sequel (1). One 75-year-old patient required manipulation for arthrofibrosis while anesthetized 3 months postoperatively. There were no deep infections postoperatively.

\section{Unilateral group}

The average age of the 24 patients in this group was 65 years (range, 32 to 82 years). Preoperative diagnoses included osteoarthritis in 21 patients, rheumatoid arthritis in 1, mixed arthritis in 1, and avascular necrosis of the femoral head in 1 . Of the 13 men and 11 women, 19 patients underwent subarachnoid block anesthesia and the remainder underwent general endotracheal anesthesia. Average surgical time ranged from 1 hour 15 minutes to 2 hours 45 minutes (average, 1 hour 55 minutes). The blood loss averaged $277 \mathrm{~mL}$, requiring transfusion in $38 \%$ of the patients in this group. An average of 0.8 unit of packed RBCs was transfused per patient.

The length of hospital stay ranged from 5 days in a patient who had a medial unicompartmental knee arthroplasty to 25 days in a patient who underwent a total knee arthroplasty and had postoperative complications of deep vein thrombosis. The average hospitalization was 10.3 days. Postoperative complications included deep vein thrombosis (1), urinary tract infections (2), and superficial phlebitis (1). There were no deep infections postoperatively.

Hospitalization costs and services, which were calculated in a manner similar to that used for the bilateral group, averaged $\$ 10,272$.

The patients were not followed up long-term. The intent of this study was to review only the incidence of perioperative complications of the two groups.

\section{Discussion}

The patients in the bilateral group were an average of 5 years older than those in the unilateral group. There was an $83 \%$ predominance of women in the bilateral group and a $48 \%$ predominance of women in the unilateral group.

The average bilateral procedure lasted 45 minutes longer than the average single, unilateral procedure. However, if two joint replacements were considered a staged procedure, then the surgical time would be 3 hours 50 minutes, which is 1 hour 13 minutes longer than for the simultaneous bilateral operations.

The patients in the bilateral group lost approximately twice as much blood as those in the unilat-
Table

Comparison of Results Between Surgical Groups

\begin{tabular}{|lcc|}
\hline & \multicolumn{2}{c|}{ Surgical groups } \\
\cline { 2 - 3 } Variables & $\begin{array}{c}\text { Bilateral } \\
\text { simultaneous } \\
\text { arthroplasties }\end{array}$ & $\begin{array}{c}\text { Unilateral } \\
\text { arthroplasty }\end{array}$ \\
\hline Age, yr & 70 & 65 \\
$\begin{array}{l}\text { Surgical time } \\
\text { Estimated blood loss, }\end{array}$ & $2 \mathrm{hr} 37 \mathrm{~min}$ & $1 \mathrm{hr} 55 \mathrm{~min}$ \\
Transfusion of packed & $419 \mathrm{~mL}$ & $277 \mathrm{~mL}$ \\
$\begin{array}{l}\text { red blood cells, U } \\
\text { Hospital days }\end{array}$ & 2.0 & 0.8 \\
Hospital cost* & 12 & 10.3 \\
\hline *Calculated in April 1987 dollar figures. & $\$ 10,272$ \\
\hline
\end{tabular}

eral group. The bilateral group received transfusions in $67 \%$ of the procedures, for an average of 2 units per person. In the unilateral group, transfusions were needed in $38 \%$ of the patients, for an average of 0.86 unit per patient.

Perioperative complications were comparable between groups. One case of deep vein thrombosis occurred in each group, and there was an equal incidence of urinary tract infections. The patient with a partial wound dehiscence had had a hematoma resulting from the administration of crystalline warfarin sodium (Coumadin), which was given for deep vein thrombosis. These conditions resolved without sequel.

The unilateral group spent an average of 10.3 days in the hospital. Based on the expected hospitalization for one joint replacement procedure, bilateral and staged (separate admission) arthroplasties would require 20 days, which would be 8 days more than for the bilateral simultaneous arthroplasties.

The average hospital cost for the bilateral group was $\$ 12,315$ compared with $\$ 10,272$ for the unilateral group. One can realize the substantial cost savings when bilateral simultaneous joint replacement arthroplasties are performed.

\section{Summary}

The results of this retrospective study support our belief that bilateral simultaneous lower extremity arthroplasties have definite advantages over bilateral lower extremity arthroplasties performed as separate staged procedures. These findings are in agreement with other authors as well. The study by Salvati and coworkers ${ }^{9}$ of 122 patients who had bilateral total hip replacements performed in one operative session (although not simultaneously) com- 
pared favorably with the bilateral total hip replacement performed in two stages, either during the same hospitalization or under two separate admissions. No increased morbidity was associated with surgical technique, postoperative care, complications, or wound healing among the group studied. The authors emphasized the impact of their data as a result of escalating surgical costs and a heightened need for bed space.

McLaughlin and Fisher ${ }^{5}$ reviewed the records of 22 patients who underwent bilateral simultaneous total knee replacements and compared the results with those of patients who had staged procedures. They found that the lowest rate of severe complications occurred in those patients undergoing bilateral simultaneous knee arthroplasties compared with those in the staged groups.

Soudry and colleagues ${ }^{11}$ described similar results from their study of 56 bilateral one-stage total knee replacements. They found an increased incidence of clinically apparent pulmonary embolism in the two-stage bilateral arthroplasty group.

Bilateral simultaneous total knee arthroplasties were found not only to be safer, but also to have demonstrated a dramatic reduction in the length of hospitalization compared with the staged procedures.

Brotherton and associates ${ }^{2}$ presented the savings for simultaneous unistaged procedures in dollars and cents referenced to December 1977 figures. They calculated a $54 \%$ savings for patients undergoing simultaneous staged procedures compared with the unilateral approach.

Most recently, Morrey and colleagues ${ }^{6}$ reviewed a total of 145 patients who underwent 290 bilateral simultaneous total knee replacements compared with 228 who had bilateral staged and 234 who had unilateral knee replacements. A total of more than 1000 knee replacements were included in the study. The authors found no significantly different risks of complications, including infection, pulmonary embolism, joint loosening, or death among the groups analyzed. Their findings provided good statistical support for performing simultaneous bilateral total knee arthroplasties.

Although the numbers in our series are low and may not be statistically significant, our results reflect the findings presented by the larger, multicenter reviews. ${ }^{2,5,6,9,11}$

With proper patient selection, experienced surgical teams, proper technique, and precautions, bilateral simultaneous lower extremity replacement arthroplasties executed in a two-team approach provide the patient with a shortened operative time and decreased length and cost of hospitalization without increased morbidity in comparison with those who undergo unilateral procedures.

1. Bracy D, Wroblewski BM: Bilateral Charnley arthroplasty as a single procedure. J Bone Joint Surg 1981;63B:354-356.

2. Brotherton S, Roberson J, Andrade J, et al: Staged versus simultaneous bilateral total knee replacement. J Arthroplasty 1986;1:221-228.

3. Gradillas EL, Volz RG: Bilateral total knee replacement under one anesthetic. Clin Orthop Related Res 1979;140:153-158.

4. Jergesen HE, Poss R, Sledge CB: Bilateral total hip and knee replacements in adults with rheumatoid arthritis. Clin Orthop Related Res 1978;137:120-128.

5. McLaughlin TP, Fisher RL: Bilateral total knee arthroplasties: Comparison of simultaneous (two-team), sequential, and staged knee replacements. Clin Orthop Related Res 1985;199:220-225.

6. Morrey BF, Adams RA, Ilstrup DM, et al: Complications and mortality associated with bilateral or unilateral total knee arthroplasty. $J$ Bone Joint Surg 1987;69:484-488.

7. Ritter MA, Randolf JC: Bilateral total hip arthroplasty: A simultaneous procedure. Acta Orthop Scand 1976;47:203-208.

8. Ritter MA, Stringer EA: Bilateral total hip arthroplasty. Clin Orthop Related Res 1980;149:185-190.

9. Salvati EA, Hughes P, Lachiewicz P: Bilateral total hip-replacement arthroplasty in one stage. J Bone Joint Surg 1978;60(S):640-644.

10. Shih C-H, Ho W-B: One-stage versus two-stage bilateral autophor ceramic total hip arthroplasty. Clin Orthop Related Res. 1985;193:141145.

11. Soudry M, Binazzi R, Insall JN, et al: Successive bilateral total knee replacements. J Bone Joint Surg 1985;67A:573-576.

12. Josefsson G, Lindberg L, Wiklander B: Systemic antibiotics and Gentamicin containing bone cement in the prophylaxis of postoperative infections in total hip arthroplasty. Clin Orthop Related Res 1981;159:194200.

13. US Bureau of the Census: Statistical Abstracts of the United States, ed 10. Washington, DC, Government Printing Office, 1987.

From the departments of orthopedics and orthopedic surgery, Botsford General Hospital, Farmington Hills, Mich.

Reprint requests to Dr Page, 28100 Grand River, Suite 209, Farmington Hills, MI 48024. 

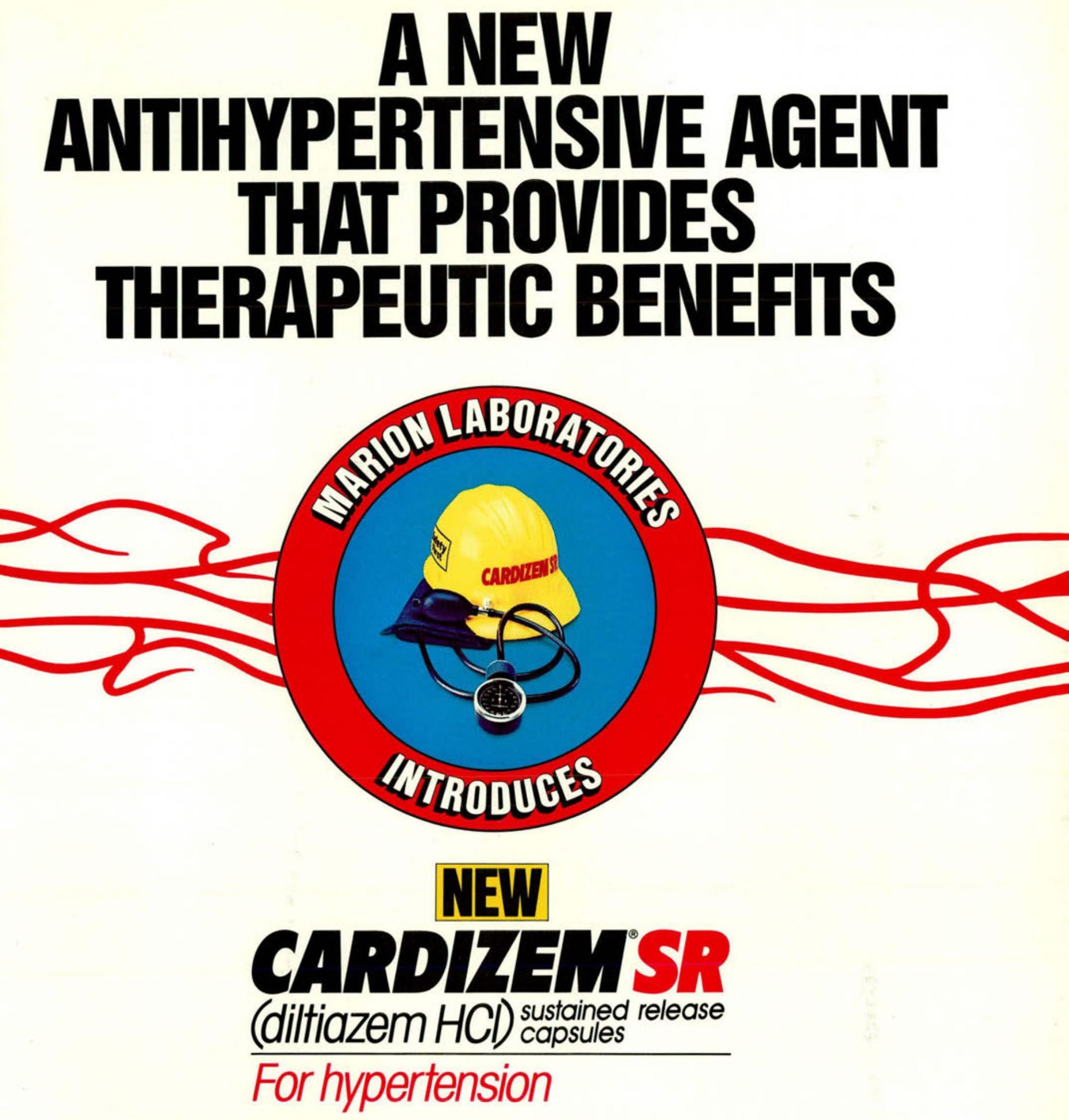

Demonstrated efficacy in a wide range of patients Low side-effect profile ${ }^{2 *}$ Convenient bid dosage

*Most commonly reported side effects in clinical trials include edema, headache, dizziness, asthenia, sinus bradycardia, flushing, and $1^{\circ} \mathrm{AV}$ block 


\section{Starting Dosage:}

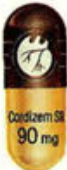 \\ 90 mg bid* \\ Also Available: 120-mg capsules}

-Dosage must be adjusted to each patient's needs, starting with 60 to 120 mg twice daily.

\section{CiRDLFUSi (diltiazem HCl) $\begin{gathered}\text { sustained release } \\ \text { capsules }\end{gathered}$}

For hypertension
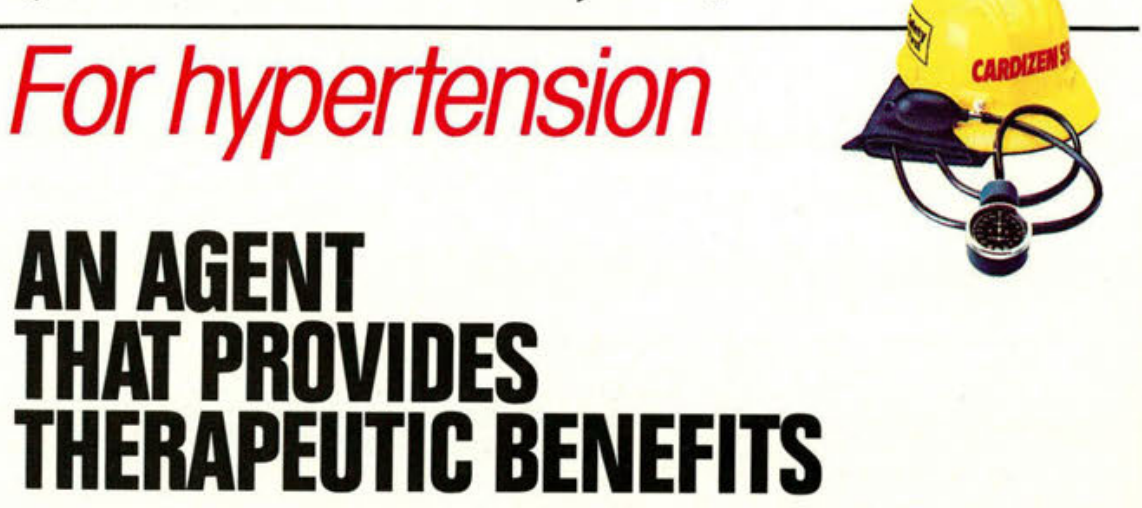

may require adjustment when starting or stopping concomitantly administered CARDIZEM to maintain optimum therapeutic blood levels

Beta-blockers: Controlled and uncontrolled domestic studies suggest that concomitant use of CARDIZEM and beta-blockers or digitalis is usually well tolerated, but avallable data are not sufficient to predict the effects of concomi-
tant treatment in patients with left ventricular dysfunction or cardiac conduction tant treatment
abnormalities

Administration of CARDIZEM (diltiazem hydrochloride) concomitantly with Administration of CARDIZEM (diltiazem hydrochloride) concomitantly with
propranolol in five normal volunteers resulted in increased propranolo levels sin

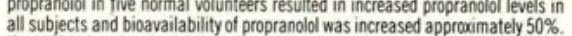
all subjects and bioavailability of propranolol was increased approximately $50 \%$. If combination therapy is initiated or withdrawn in conjunction with propranolol
an adjustment in the propranolol dose may be warranted. (See WARNINGS.) an adjustment in the propranolol dose may be warranted. (See WARNNINGS)
Cimetidine: A study in six healthy volunteers has shown a significant increase in peak diltiazem plasma levels $(58 \%)$ and area-under-the-curve $(53 \%)$ atter 1-week course of cimetidine at $1,200 \mathrm{mg}$ per day and diltiazem $60 \mathrm{mg}$ per da. Ranitidine produced smaller, nonsignificant increases. The effect may be me. diated by cimetidine's known inhibition of hepatic cytochrome P-450, the enzym system probably responsible for the first-pass metabolism of diltiazem. Patients currently receiving diltiazem therapy should be carefully monitored for a change in pharmacological effect when initiating and discontinuing therapy with cimetidine. An adjustment in the diltiazem dose may be warranted

Digitalis: Administration of CAROIZEM with digoxin in 24 healthy male subjects increased plasma digoxin concentrations approximately $20 \%$. Another investigator found no increase in digoxin levels in 12 patients with corronary artery disease. Since there have been conflicting results regarding the effect of digowin levels, it is recommended that digoxin levels be monitored when initiating. adjusting, and discontinuing CARDIZEM therapy to avoid possible over- of under-digitalization. (See WARNINGS

Anesthetics: The depression of cardiac contractility, conductivity, and automaticity as well as the vascular dilation associated with anesthetics may be potentiated by calcium channel blockers. When used concomitantly, anesthetics and calcium blockers should be titrated carefully

Carcinogenesis, Mutagenesis, Impairment of Fertility. A 24-month study in rats and a 21 -month study in mice showed no evidence of carcinogenicity. There rats and a 21-month study in mice showed no evidence of carcinogenicity. There
was also no mutagenic response in in vitro bacterial tests. No intrinsic effect on was also no mutagenic response
fertility was observed in rats.

Pregnancy. Category C. Reproduction studies have been conducted in mice, Pregnancy. Category C. Reproduction studies have been conducted in mice,
ratss and rabbits. Administration of doses ranging from five to ten times greater rats, and rabbits. Administration of doses ranging from five to ten times greater
(on a mg/kg basis) than the daily recommended therapeutic dose has resulted in (on a mg/kg basis) than the daily recommended therapeutic dose has resulted in cause skeletal abnormalities. In the perinatal postnatal studuies, there was some reduction in early individual pue werights and survival rates. There was an reduction in early individual pup weights and survival rates. There was an
increased incidence of stillibirths at doseses of 20 times the human dose or greater. increased incidence of stillitrths at doses of 20 times the human dose or greater. There are no well-controlled studies in pregnant women; therefore, use
CAROIZEM in pregnant women only if the potential benefit iustifies the potential CARolzem in preger

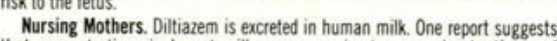
that concentrations in breast milk may approximate serum levels. If use of CAROIZEM is deemed essential, an alternative method of infant feeding should be instituted.

Pediatric Use. Saftety and effectiveness in children have not been established. ADVERSE REACTIONS

Serious adverse reactions have been rare in studies carried out to date, but it should be recognized that patients with impaired ventricular function and cardiac conduction aunormaitites have usualiy been excluded from these studies.

The adverse eventis described below represent events observed in clinical studies

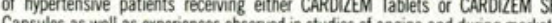
Capsules as well as experiences observed in studies of angina and duning marketing. He most com enents in herension studes are shown in a table with tates in placebo patients stiown for companison. Less common events are listed by body system, these include any adverse reactions seen in angina studies that were not coserved in hipentension studies. In all hypertensive patients studied lover

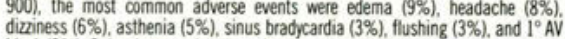
dizoness $(3 \%)$. Onily edema and perhaps bradcardia and diziness were dose related The most common events observed in clinical studies (over 2.100 patients) of angina patients and hypertensive patients receiving CAROIZEM Tablets or CARDIZEM SR Capsules were (ie, greater than $1 \%$ ) edema (5.4\%), headache (4.5\%), diziness (3.4\%), asthenia $(2.8 \%)$, first-degree Av block (1.8\%), flushing (1.7\%), nausea $(1.6 \%)$, bradycardia (1.5\%), and rash (1.5\%).

\begin{tabular}{|c|c|c|}
\hline \multicolumn{3}{|c|}{$\begin{array}{l}\text { DOUBLE BLIND PLACEBO CONTROLLED } \\
\text { HYPERTENSION TRIALS }\end{array}$} \\
\hline Adverse & $\begin{array}{l}\text { Diltiazem } \\
N=315 \\
\# \text { pts }(\%) \\
\end{array}$ & $\begin{array}{l}\text { Placebo } \\
N=211 \\
\text { \# pts } 3 \%\end{array}$ \\
\hline headache & $38(12 \%)$ & $17(8 \%)$ \\
\hline AV block first degree & $24(7.6 \%)$ & $4(1.9 \%)$ \\
\hline diziness & $22(7 \%)$ & $6(2.8 \%)$ \\
\hline edema & $19(6 \%)$ & $2(0.9 \%)$ \\
\hline bradycardia & $19(6 \%)$ & $3(1.4 \%)$ \\
\hline ECG abnormality & $13(4.1 \%)$ & $3(1.4 \%)$ \\
\hline asthenia & $10(3.2 \%)$ & $1(0.5 \%)$ \\
\hline constipation & $5(1.6 \%)$ & $2(0.9 \%)$ \\
\hline dyspepsia & $4(1.3 \%)$ & $1(0.5 \%)$ \\
\hline nausea & $4(1.3 \%)$ & $2(0.9 \%)$ \\
\hline palpitations & $4(1.3 \%)$ & $2(0.9 \%)$ \\
\hline polyuria & $4(1.3 \%)$ & $2(0.9 \%)$ \\
\hline somnolence & $4(1.3 \%)$ & - \\
\hline alk phos increase & $3(1 \%)$ & $1(0.5 \%)$ \\
\hline hypotension & $3(1 \%)$ & $1(0.5 \%)$ \\
\hline insomnia & $3(1 \%)$ & $1(0.5 \%)$ \\
\hline rash & $3(1 \%)$ & $1(0.5 \%)$ \\
\hline AV block second degree & $2(0.6 \%)$ & - \\
\hline
\end{tabular}

In addition, the following events were reported infrequently (less than 1\%) or have been observed in angina trials. In many cases, the relation to drug is uncertain.

Cardiovascular: Angina, arrhythmia, bundle branch block, tachycardia, ventricular extrasystoles, congestive heart failure, syncope.

Nervous System: Amnesia, depression, gait abnormality, hallucinations, nesvousness, paresthesia, personality change, tinnitus, tremor. vousness, paresthersial dreams.

Gastrointestinal: Anorexia diarthea, dysgeusia, mild elevations of SGOT, SGPT, and LDH (see hepatic warnings), vomiting, weight increase, thirs:

Dermatological: Petechiae, prunitus, photosensitivity, urticaria

Other: $\quad$ Amblyopia, CPK increase, dyspnea, epistaxis, eye irritation, hyperglycemia, sexual difficulties, nasal congestion, nocturia.

The following postmarketing events have been reported infrequently in patients receiving CARDIZEM: alopecia, gingival hyperplasia, erythema multiforme, and leukopenia. Definitive cause and effect relationship between these events and CARDIZEM therapy cannot yet be established.

Issued 1/89

References: 1. Pool PE, Seagren SC, Salel AF: $A m$ J Cardiol 1985;56:86H-91H. 2. Frishman WH, Zawada ET Jr, Smith LK, et al: Am J Cardiol 1987;59:615-623.

Another patient benefit product from 